\title{
Análisis de la visibilidad de las series de televisión en la Web
}

\author{
Por Elena De-la-Cuadra-Colmenares y María-Victoria Nuño-Moral
}

\begin{abstract}
Resumen: Se analiza la organización y presentación de los espacios web que las principales cadenas de televisión nacional dedican a las series de televisión. Se centra en una doble perspectiva: por un lado reflejar las estructuras y contenidos de cada sitio web y, por otro, enunciar las opciones disponibles para el usuario. Para su realización se ha tomado como referencia la propuesta realizada por Codina, Aubia y Sánchez.(2008) y se ha elaborado una plantilla de trabajo basada en el examen de aspectos cualitativos y cuantitativos.

Palabras clave: Internet, Visibilidad web, Televisión, Series de televisión, Documentación audiovisual.

Title: Analysis of the Web visibility of television series

Abstract: The organization and presentation of web spaces that the main national television channels dedicate to television series are analyzed. We focus the study on a double perspective, reflecting on one hand the structure and content of each web site and, on the other, the options available to the user. The proposal of Codina, Aubia and Sánchez (2008) is taken as a reference for methodology and a working template has been developed based on the review of both qualitative and quantitative aspects.
\end{abstract}

Keywords: Internet, Web visibility, Television, Television series, Audiovisual documentation.

De-la-Cuadra-Colmenares, Elena; Nuño-Moral, MaríaVictoria. "Análisis de la visibilidad de las series de televisión en la Web". El profesional de la información, 2010, noviembre-diciembre, v. 19, n. 6, pp. 658-666.

DOI: $10.3145 /$ epi.2010.nov.13

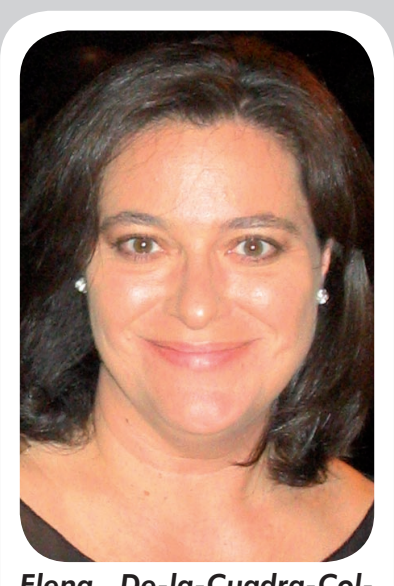

Elena De-la-Cuadra-Colmenares es licenciada en comunicación audiovisual por la Universidad Complutense de Madrid y doctora en documentación. Ha simultaneado su carrera docente con el trabajo en empresas audiovisuales, tanto en producción como en documentación (por ejemplo, como documentalista en Cuéntame cómo pasó y en diversas productoras). Su tesis doctoral abordó la relación entre producción audiovisual y nuevas tecnologías.

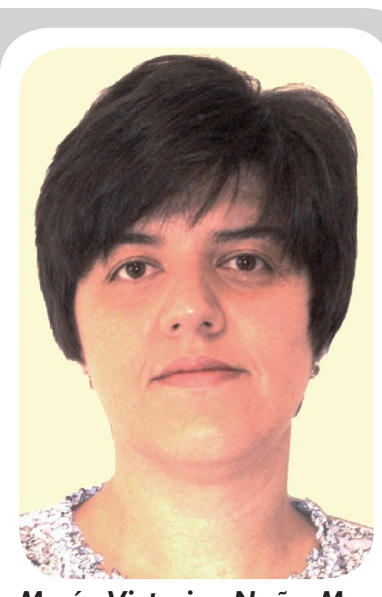

María-Victoria Nuño-Moral es profesora titular de la Fac. de Biblioteconomía y Documentación de la Univ. de Extremadura, donde imparte documentación informativa y audiovisual en las licenciaturas de documentación y comunicación audiovisual. Autora de diferentes publicaciones, se ha especializado en el ámbito de los medios de comunicación, participando en cursos, congresos y proyectos de investigación.

\section{Introducción}

LAS CADENAS DE TELEVISIÓN emplean sus sitios web con una finalidad primordialmente periodística. Sin embargo, ofrecen otros productos audiovisuales (ficción, divulgación, concursos, etc.) y apuestan, en un primer momento, por exponer gran parte de su material en la Red ${ }^{1}$. Toman como punto de partida los formatos tradicionales pero las opciones se diversifican y el usuario cuenta con un número mayor de servicios. Así, ofrecen los denominados servicios de valor añadido (SVA) y proporcionan nuevas cuotas de información imposibles anteriormente ${ }^{2}$.
Tal y como señalan Jiménez, Fuentes y González (2003) "los medios de comunicación en internet están en disposición de aprovechar las potencialidades que ofrece tanto la tecnología web como las propiedades de la información digital. Este aprovechamiento se ha traducido en una serie de características que definen el perfil de los medios digitales y los diferencian de los analógicos: interactividad, integración de información multimedia, superación de la periodicidad, inmediatez informativa, personalización de contenidos".

Este estudio se centra en la información que las cadenas de televisión generalistas de España ofrecen en internet, concretamente sobre sus series, considerando que es uno de los productos que más audiencia puede generar ${ }^{3}$. Junto con las retransmisiones deportivas, acaparan los primeros puestos de los rankings de telespectadores $^{4}$ (Stewart, 2001). En España, series como Farmacia de guardia (Globomedia, 1991-1995), Médico de familia ${ }^{5}$ (GlobomedialEstudios Picasso, 1995-1999) o Cuéntame cómo pasó ${ }^{6}$ (Grupo Ganga, 2001-) han estado presentes en las listas de los programas más vistos.

Hay que matizar algunos aspectos sobre el concepto de "serie de televisión". Tal y como han reco- 
nocido algunos autores (Dafonte, 2010), resulta difícil definir claramente los géneros televisivos. En la eterna discusión género/formato, Inmaculada Gordillo (2009) divide los productos televisivos en géneros, formatos y programas. Así, los géneros serían: informativo, ficcional, docudramático, publicitario y entretenimiento. Las series, además, han evolucionado a lo largo de los años. Hemos asistido a muchos cambios en el formato: hibridaciones, derivaciones, acercamiento a otros formatos (por ejemplo, a otros más realistas, García-De-Castro, 2008). Como afirman GómezMartínez y García-García (2010), "hoy vivimos un continuo mestizaje de géneros [...]. Los formatos de televisión se mantienen con una cierta rigidez, y sólo eventualmente alguna afortunada combinación permite reclasificar una determinada serie en un punto intermedio entre dos formatos reconocibles".

Por ello vamos a tratar de definir el formato "serie", marcando su diferencia con otros similares, para acotar el objeto del análisis. Consideraremos "serie tradicional" a aquella en que el punto de partida son unos personajes fijos en un escenario, pero en cada episodio se tratan temas diferentes y se unen personajes distintos en escenarioas diversos, incluyendo exteriores (por ejemplo, los casos que resuelven unos policías, o la sala de urgencias de un hospital). Unos personajes son fijos y otros intervienen sólo en un episodio. Ejemplos: El comisario (BocaBoca Producciones, 1999-2009; Hospital central, (Videomedia/Estudios Picasso, 2000-)... Así, diferenciamos este formato (objeto de este trabajo) de otros semejantes:

- el culebrón o serial (donde la acción afecta una y otra vez a los mismos personajes, casi siempre en circunstancias dramáticas, y en los mismos escenarios, sin nuevas inclusiones ni de personajes ni de decorados), por ejemplo Cristal
(Venezuela, RCTV, 1985) o Abigail (Venezuela, $R C T V$, 1988);

- las sit-com o "comedia de situación" (en las que el peso del formato recae en el gag, en la comicidad de las situaciones, y en las que decorados y personajes son escasos, incluso los movimientos de cámara son siempre los mismos). Ejemplos: Cheers (Charles/Burrows/Charles Productions, 19821993); Friends (Warner Bros Television, 1994-2004);

- las que aquí denominamos camera-com (series del estilo de Camera Café (Magnolia, 20052009), en las que la cámara está fija en un punto del plató, y los personajes son los que se acercan o alejan de ella);

- o miniserie (pocos episodios, heredera del largometraje cinematográfico).

\section{"Los medios digitales se convierten en fuente de información que posibilita el acceso instantáneo y en cualquier momento al contenido"}

\section{Propuesta de parámetros de análisis}

Se plantea aquí una serie de parámetros que atienden a las necesidades informativas del usuario, teniendo en cuenta que cualquier persona puede acceder a las webs de las series buscando una respuesta. La cantidad y la calidad de información aportada es lo que se valora con dicha cuantificación. Esta propuesta considera la idea expresada por Codina (2004): "Los parámetros responden a la pregunta: ¿qué queremos evaluar? Las propiedades de un recurso son casi ilimitadas. Conviene seleccionar qué examinamos. [...] Por otro lado, los sitios web representan una amplia variedad de géneros $[\ldots]$ Cada uno de estos géneros, para ser evaluados, requiere parámetros específicos"?.

A continuación, se establecen los elementos que se han valorado en las cadenas analizadas y que constituyen el esquema completo del estudio realizado.

\section{Página principal de la cadena}

Examen de la web, sin entrar en ningún enlace. Se analiza:

1.1. Acceso claro al apartado Series: se observa si se permite un acceso sencillo y rápido a las series, sin necesidad de pasos intermedios.

1.2. Presencia de series en el menú carrusel: se llama menú carrusel a la sucesión de fotografías y texto que aparecen en la página principal, ocupando una parte mayoritaria de la pantalla, en el que las informaciones se suceden cada pocos segundos. Se emplea como publicidad de series, programas o novedades de la cadena. En caso de que el usuario quiera retroceder o avanzar, puede seleccionar qué parte del carrusel es la que se quiere ver.

1.3. Acceso destacado a alguna serie: cuando una cadena quiere promocionar una serie o ésta tiene muchos seguidores, desde la página principal ya se puede acceder a varias cosas: blogs, artículos, resúmenes, chats, merchandising, vídeos, etc.; fue el caso de Perdidos ( $A B C$, 2004-2010), especialmente cuando se acercaba el final.

\section{Página de series}

El segundo nivel de acercamiento a los contenidos es la página que el sitio dedica a las series, a todas en general. Se valora:

2.1. Presencia de vídeo: como veremos en otros apartados, el hecho de que un vídeo esté insertado en la página no es siempre positivo. Muchos usuarios, especialmente en vacaciones, utilizan una conexión a internet portátil; es decir, utilizan un módem USB y el coste total del 
servicio depende de la cantidad de $\mathrm{KBs}$ que circulen. En muchos casos el usuario navega procurando no acceder a archivos pesados. Por tanto debería haber una opción de descarga de manera que no comenzase automáticamente.

2.2. Presencia o no de spoilers ${ }^{8}$. En los foros se habla de un spoiler cuando alguien cuenta algún aspecto de la trama de una serie antes de que se haya emitido. Las propias revistas de televisión, las autopromociones de las cadenas, incluso las páginas oficiales de las series, en muchas ocasiones, desvelan aspectos que sucederán en un futuro. Esto no siempre es positivo: hay muchos espectadores que prefieren ver la serie sin saber qué va a ocurrir, y así respetar la línea temporal pensada por los guionistas, y disfrutar de cada momento sin adelantar acontecimientos.

2.3. Presencia predominante de series actuales: parece evidente que el usuario que entra en la web de una cadena para buscar información sobre series, lo que quiere ver son aquellas que se están emitiendo en ese momento. Por eso sería positivo que éstas ocuparan un lugar preferente dentro de la página y así evitar una cierta confusión en el espectador.

2.4. Enlaces a series pasadas: pese a que el espacio preferente, como decíamos, lo deberían ocupar las series presentes, la cadena debería tener un espacio dedicado a las series pasadas, especialmente a las de producción propia. El espacio de almacenamiento ya no es un problema, y tendría que ser posible el acceso a todas las que hayan sido emitidas. Cuando el espacio de almacenamiento sí sea un problema, deberían quedar visibles, al menos, las emitidas en los últimos cinco años.

2.5. Enlaces a series futuras. Sería un valor añadido ofrecer información sobre las series que está preparando la cadena, si es producción propia, o de aquellas cuyos derechos haya adquirido, si son com- pradas. Sería una buena manera de enganchar al espectador, especialmente si la página está actualizada adecuadamente (por ejemplo, con la fecha de estreno, y no el incierto "próximamente").

2.6. Enlaces a series que en realidad son otros formatos: algunas cadenas incluyen entre las series formatos que no son tal cosa. Algunos de ellos son novedosos, otros no, pero aparecen junto a las series. Es conveniente diferenciar una serie de una sit-com, por ejemplo.

2.7. Menú carrusel: como en la página principal, puede haber un carrusel promocional con las series más actuales, ofreciendo información sin caer en el spoiler.

\section{Página de una serie en concreto}

Una vez que el usuario ha accedido a la página de una serie, se valoran los siguientes puntos:

\subsection{Acceso a vídeos:}

- vídeo presente sin intervención del usuario,

- vídeo con el avance del próximo capítulo,

- vídeos breves con momentos clave de determinados capítulos,

- vídeos de las temporadas anteriores.

Como hemos visto en las páginas previas, no es positivo que aparezcan vídeos sin la petición del usuario. Naturalmente, si los necesita, debería poder disponer de ellos.

\subsection{Información sobre:}

- la trama,

- los actores,

- los personajes,

- el equipo técnico,

- corporativa: año de creación, productora, inicios, intenciones, resumen brevísimo de la evolución de los personajes a lo largo de las temporadas,

- día y hora de emisión.
La información es el punto fuerte de esta parte de la web. Hasta ahora, en los niveles anteriores, la navegabilidad y el diseño ocupaban el núcleo del análisis; ahora es fundamental que el usuario encuentre todos esos datos.

En el caso de que la serie sea un spin off de otra serie anterior (una nueva serie que toma parte de los personajes y de las tramas de una serie anterior: por ejemplo, Aída es un spin off de 7 vidas), es fundamental mencionarlo y ofrecer la información necesaria que vincule a ambos productos.

3.3. Enlace a los sitios web de series internacionales: cuando una serie haya sido comprada a una productora, siempre que tenga web oficial, debe estar presente el enlace a la serie y a la productora. Ha de incluirse un aviso de que el usuario va a abandonar la web de la cadena, y de que puede encontrar spoilers en la página de destino.

\section{Actualización}

4.1. Agilidad en la información sobre el cambio de horario, retrasos, cambio de día, supresión de un capítulo una semana, etc. Es fundamental que se mantenga actualizado al minuto el cambio de horario de las series. Dada la mala costumbre de la contraprogramación, quizá es pedir imposibles (eso es precisamente la contraprogramación, no saber hasta el último momento qué va a emitir la cadena).

\section{Comunidad virtual}

Dentro de este bloque se examina la necesidad y presencia de:

- registro previo,

- foros de la serie,

- foros sobre capítulos emitidos,

- foros sobre capítulos simultáneamente a la emisión,

- chat simultáneo a la emisión,

- concursos, sorteos, envío de comentarios, de fotos... 
El registro previo a la participación en los foros de las webs es una manera de evitar intervenciones exageradas, ofensivas o fuera de lugar; con esta práctica se puede ejercer un cierto control sobre el usuario (además de conservar su correo electrónico para posibles envíos de publicidad o promociones). Pero en ocasiones rechaza registrarse por la molestia (especialmente si debe completar demasiados datos personales) y por el tiempo necesario para hacerlo.

Es positivo que exista un foro para cada serie, así como para determinados capítulos. El chat en directo, simultáneo a la emisión, es una interesante forma de saber el nivel de implicación de los usuarios con la serie.

El envío de fotografías o de otro tipo de archivos, especialmente si hay un sorteo, anima la página y aporta contenidos.

\section{"Los logros experimentados en la Red por la televisión son incalculables desde la perspectiva del usuario y los contenidos"}

\section{Merchandising}

6.1. Productos con la estética de la serie.

6.2. Productos no relacionados directamente con la serie.

En el segundo punto nos referimos a productos que no son exclusivamente de la serie, pero que quizá identifican a un personaje o una situación (por ejemplo, unas gafas de sol como las de Grissom, personaje de $C S I$ ).

\section{Interacción}

7.1. Correo de la página de series.
7.2. Correo de la página de cada una de las series.

$\mathrm{Al}$ margen de lo establecido en el punto 5, el correo debe permitir que el usuario consiga algún tipo de información sobre la serie que la página no facilita. Es fundamental que el correo sea contestado antes de 48 horas.

\section{Estudio de casos}

La investigación que aquí se expone tiene una doble finalidad. Por un lado, conocer cuál es la situación actual en la que se encuentran las series de televisión en internet así como los contenidos, servicios y opciones que aportan mediante sus sitios web; y por otro, realizar una comparación basada en los resultados obtenidos.

Primer indicador: análisis de la página principal de la cadena en relación con las series.

En la home de las diferentes cadenas, el usuario cuenta con un menú principal y con una opción claramente identificada dedicada a Series, excepto en el caso de La sexta donde el acceso se realiza desde Programas. Tanto Antena 3 como Cuatro cuentan con un menú carrusel en el que encontramos informaciones sobre series en emisión o cuyo estreno ocurrirá próximamente. Antena 3 y TVE destacan en esa página general (al margen del menú carrusel) alguna de sus series en emisión o cuyo estreno esté próximo como es el caso de Las chicas de oro (TVE). Telecinco muestra información de alguna de sus series pero no lo hace en un lugar destacado.

Segundo indicador: análisis de la página principal de series

Telecinco no cuenta con una página general de series sino que desde el sitio web de la cadena y mediante el menú Series, se puede acceder al conjunto de capítulos íntegros de todas ellas contando además con la posibilidad de ver en primicia y alta calidad algunos mediante la opción Preestrenos de Telecinco.es. $\mathrm{O}$ bien, posibilita ir directamente a cada una de las páginas destinadas a cada serie de manera individualizada. Esta oportunidad de ver todos los capítulos completos de las diferentes temporadas está presente igualmente en toda la muestra de televisiones analizada.

Cabría destacar en este caso un cierto desorden en la organización y estructuración del binomio menú/contenidos ya que mientras que desde su página principal, dentro del menú Series incluye la opción Otros destinada, en principio, a series fuera de emisión, se puede observar cómo algunos casos que ya están fuera de pantalla disponen de un acceso directo desde el menú Series. Cuando entramos en algún caso concreto, la barra de texto permanece pero la ordenación y cantidad de series ofrecidas varía; esto ocurre, por ejemplo, si observamos el acceso a Acusados. Además, desde dicha barra se mezclan series con otros formatos, tal es el caso de Camera Café. La inclusión de formatos que se alejan de las series tradicionales los encontramos en Cuatro con La isla de los nominados o en La sexta con La tira.

Telecinco incluye un acceso a $T V$ movies, siendo éstas películas hechas para televisión basadas en un hecho reciente o histórico que la cadena emite de manera fragmentada. El concepto de TV movies difiere del de Telefilm en que éste se utilizaba para películas de poca calidad, bajo un argumento previsible, que se emitían los fines de semana con una función principalmente de relleno de la emisión. Por su parte, las TV movies aportan un matiz de actualidad o de retrato histórico como se refleja en ejemplos tales como La duquesa (Ficción media, 2010) o Paquirri (Ficción media, 2010). En cualquier caso su génesis es cinematográfica aunque se emiten como miniseries en días o semanas sucesivos. 
En cuanto a la organización de la página, en el otro extremo se situaría TVE al distinguir visiblemente las series que están en antena de aquellas que ya están fuera de emisión. En concreto muestra enlaces a las diferentes series clasificadas por: Series en emisión, Próximamente, Temporadas completas y Series literarias.

A excepción de Cuatro que cuenta con la presencia de vídeos, opción disponible para todas sus series, donde el usuario decidirá sobre su visualización o no, el resto de casos analizados no dispone de dicha elección.

En general predominan las series actuales aunque también se incluye el anuncio de próximos estrenos con enlace incluso a sus sitios web tal como Hispania. La leyenda (Bambú producciones, 2010) o ¿Dónde está Elisa? (Film in Florida, 2010) (ambas en Antena 3), cuyo estreno tuvo lugar el 16 de agosto. Hemos observado, igualmente la existencia de enlaces a series pasadas aunque, tal y como hemos mencionado anteriormente, sería conveniente que se tuviera en cuenta su finalización a la hora de organizar la web estableciendo, por ejemplo, un apartado o enlace donde se especifique públicamente su estado. En algunos casos, en un mismo nivel se incluyen series que ya han finalizado, tal y como ocurre con Flashforward (HBO/ ABC, 2009) o Perdidos (Cuatro).

En relación con este último aspecto, La sexta presenta sus series mediante un listado a partir del cual, el usuario puede acudir a cada web u obtener una breve descripción argumental y técnica de la serie. Hay que destacar la necesidad de homogeneizar su información dado que mientras en el frame situado en la página general de la cadena donde se mencionan los sitios web que la integran, el dedicado a series está actualizado, el listado disponible en la página general de series dista de dicha realidad.

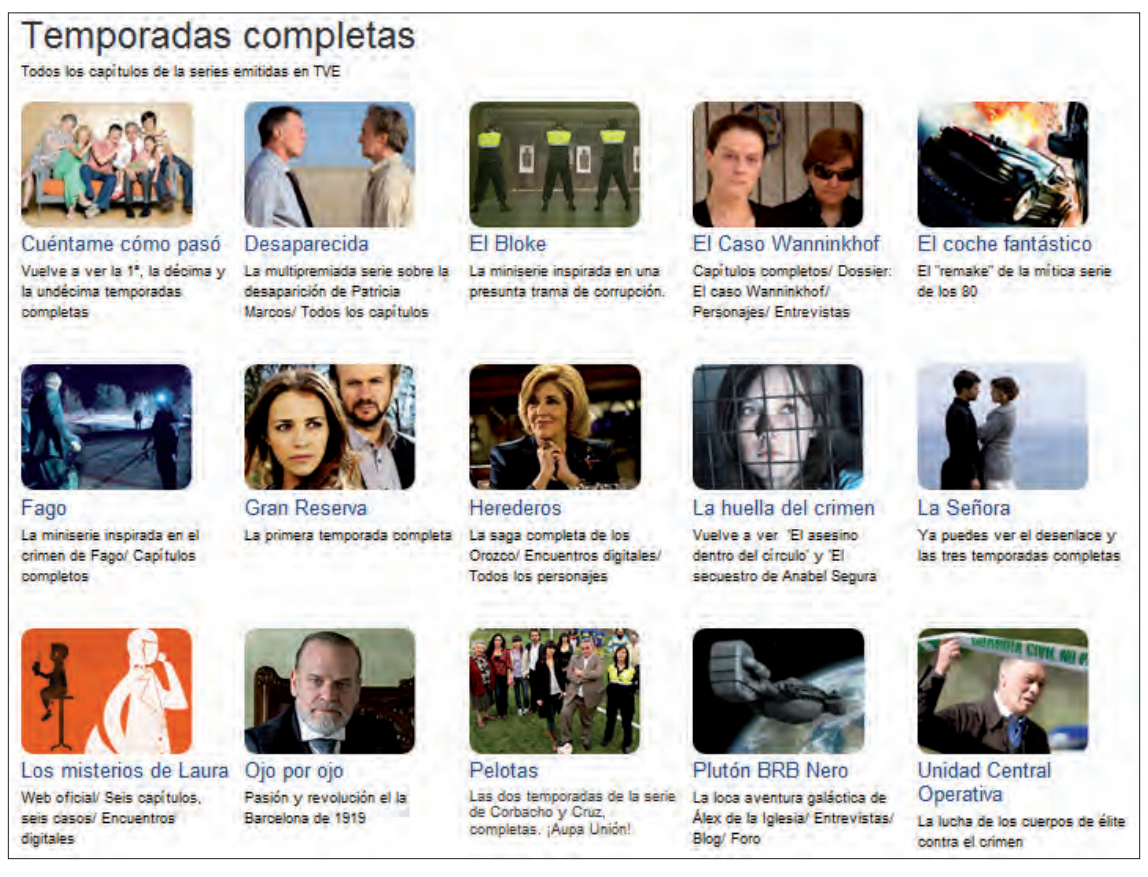

Figura 1. Acceso al archivo de series ya emitidas en TVE, http://www.rtve.es/television/series-tve/

Un ejemplo curioso lo encontramos con la serie NAVY: investigación criminal (CBS/Belisarius production, 2003). Está presente en el listado mencionado anteriormente pero desde él simplemente se accede a su descripción y no se puede acceder a su página, la cual sí está disponible desde el frame dedicado a "Series recomendadas" cuando nos situamos en la web de otra serie, por ejemplo Bones. Este hecho pone de manifiesto, una vez más, la necesidad de revisar y actualizar la información vertida por medio de los sitios web corporativos.

\section{"Las series de ficción son uno de los productos que más audiencia puede generar junto con las retransmisiones deportivas"}

Para concluir con este segundo parámetro indicar que ninguno de los casos dispone de menú carrusel en la página general de series y en cuanto a los spoilers, únicamente se ha encontrado un ejemplo en $\mathrm{Cua}$ tro, en relación con la serie House donde se revelan datos acerca de la séptima temporada. Lo mencionamos dentro de este bloque ya que el usuario dispone de dicha información desde la página principal desde el blog de la serie. Obviamente, también está disponible desde la página individual establecida para ella.

\section{Tercer indicador: análisis de la pá- gina concreta de series}

$T V E$ es el caso que cuenta con todas las opciones informativas audiovisuales dado que ofrece el vídeo del último y próximo capítulo, así como de los mejores momentos y capítulos íntegros de cada temporada.

Por su parte, Antena 3 y Telecinco cuentan igualmente con todas las opciones excepto con el adelanto del próximo capítulo. En el caso de Antena 3, los vídeos no se encuentran en una zona destacada de la página sino en pequeños frames situados en la parte de noticias; un ejemplo claro lo vemos con una de las series de mayor audiencia de la cadena: El internado (Globomedia, 2007-). Telecinco sí cuenta con la opción "Momentos clave" aunque sólo se hace referencia al último capítulo. Esta posibilidad puede inducir a error con otra existente en esta 


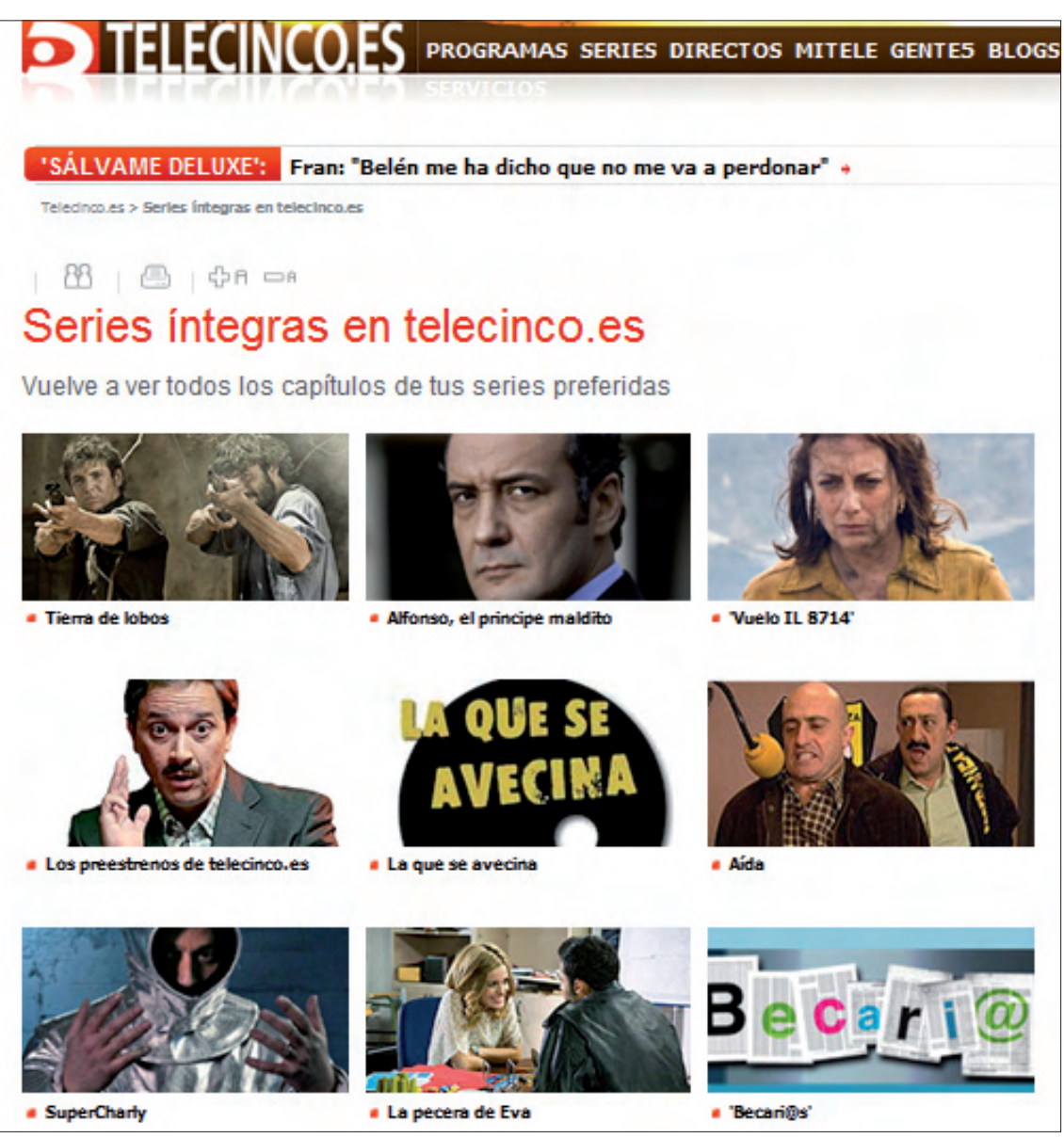

Figura 2. Series de TV de Telecinco, http://www.telecinco.es/

misma página como es Claves pero en este caso, la información aportada es textual tal y como se explica más adelante.

En Cuatro, en el vídeo promocional que aparece de cada serie hay un enlace a su correspondiente web. Encontramos, principalmente, vídeos basados en autopromociones de las distintas series. En algunos casos el usuario cuenta con momentos destacados en algunas de ellas tal y como ocurre con Anatomía de Grey (Touchstone/ABC, 2007-).

Mención aparte merece La sexta ante la ausencia total de vídeos. Esta web basa toda su información en texto y fotografías. En relación con este último material, conviene resaltar que todas las cadenas cuentan con galerías de fotos.

En lo que a información textual se refiere, la falta de uniformidad es una característica que encontramos en las diferentes cadenas, tanto en su conjunto como individualmente. a modo de punto de partida, pero no del transcurrir de la historia.

La explicación argumental también está presente en todas las series de TVE excepto en Los Tudor (Peace Arch Entertainment Group, 2007), y en La sexta donde se ofrece igualmente información relacionada con capítulos de otras temporadas. Se puede decir que es esta cadena la que aporta mayor uniformidad en la plantilla utilizada para presentar la información en cada página individual. No obstante, mientras que el resto de cadenas analizadas utilizan la opción Capítulos para visualizar los episodios, en este caso, ofrece información textual sobre cada uno de ellos. Hay que recordar que $L a$ sexta no cuenta con ningún vídeo.

Todas cuentan con información sobre los personajes y en Antena 3 y Telecinco también sobre los actores. En Cuatro no existe uniformidad a este respecto ya que mientras en series como Perdidos o Anatomía de Grey se dan explicaciones sobre actores, personajes y el rol que desempeñan, en otros casos como en House sólo disponemos de una breve presentación de los personajes cuyo texto viene acompañado de diferentes fotografías.

Telecinco, Cuatro y TVE ofrecen datos sobre el día y hora de emisión aunque es necesaria su revisión en algunos casos. Por ejemplo, en la web de la serie Life se incluye un horario de emisión que en la actualidad está ocupado por otra serie: NCIS Los Ángeles (CBS, 2009). Por su parte, TVE, desde su página general de series, informa del día y hora de emisión así como de los capítulos disponibles correspondientes a las distintas temporadas.

Como se ha mencionado en otro apartado, la serie House (NBC/Universal, 2004) (Cuatro) es la única que cuenta con el adelanto de momentos que podremos ver en la próxima temporada y en dicho spoiler, precisamente se advierte de la presencia de datos nuevos sobre la trama. 
Finalmente mencionar que no es habitual proporcionar la fecha de creación o emisión de manera clara (sólo se ha encontrado en la serie Amar en tiempos revueltos (Diagonal TV, 2005) de TVE. Tampoco es usual disponer de la ficha artística y técnica de las series; sólo se ha encontrado algún ejemplo en $T e$ lecinco-Acusados- y TVE-Amar en tiempos revueltos y Herederos(Quarzo, 2008-).

Mención especial merece la serie Amar en tiempos revueltos ya que se puede seguir en directo desde la Red coincidiendo con la emisión tradicional. Además, es el único caso donde se muestra de manera explícita información corporativa referida a "inicios e intenciones". Sirva de muestra el siguiente párrafo localizado en su web: "Amar en tiempos revueltos retrata con una fidelidad extraordinaria y con gran atención al detalle cuál fue la situación personal y social de las personas que vivieron y sufrieron las consecuencias de la guerra".

En relación con el envío a las páginas oficiales cuando se trata de series extranjeras hay que mencionar que no se ha hallado ningún ejemplo en los casos analizados; todas se mantienen en la web de la cadena.

\section{Cuarto indicador: actualización}

Dado que se ha aplicado el término "navegar" al hecho de que un usuario consulte páginas web, se puede aprovechar la metáfora del mar para indicar que, en las webs de televisión, cuanto más nos adentramos en las profundidades, menos luz hay. Parece que hay páginas olvidadas, que en su día ofrecían información sobre novedades de alguna serie, y que siguen predicando en el desierto novedades y horarios que hace tiempo que ya no son.

Es particularmente difícil evaluar este parámetro en los meses de verano (cuando se ha realizado este estudio), ya que las emisiones

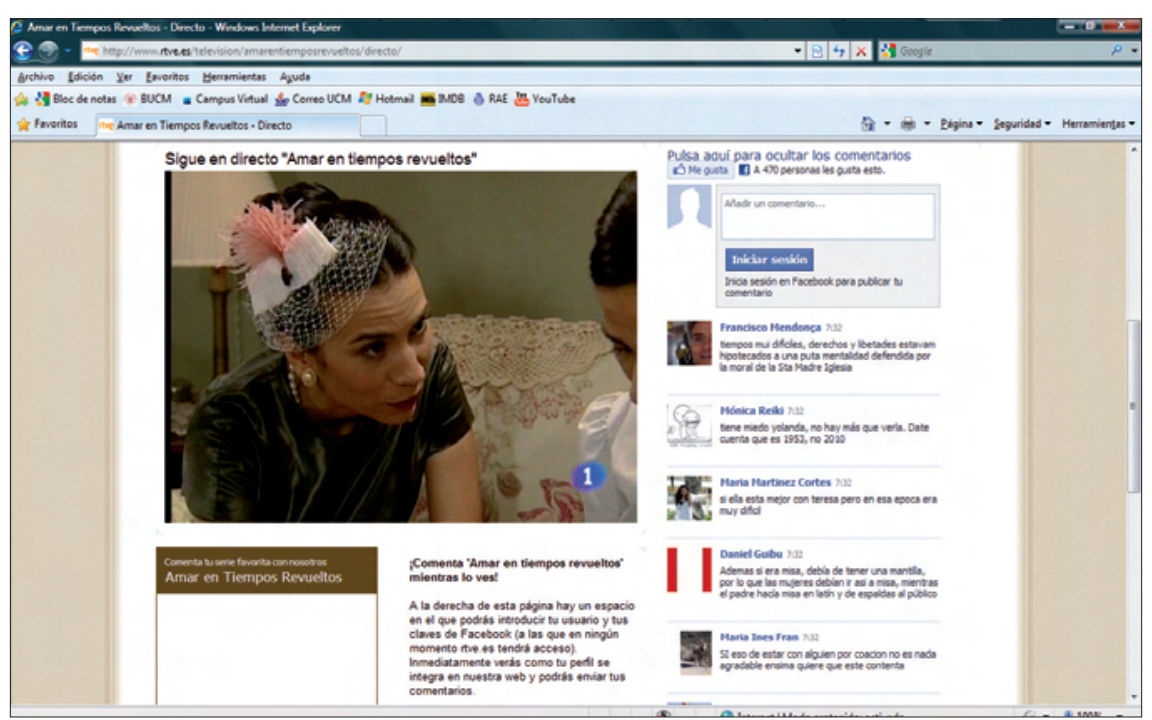

Figura 3. Chat de Facebook durante la emisión de la serie Amar en tiempos revueltos (TVE) en televisión son, en su mayoría, repeticiones, y la información de las webs se refiere a la emisión de capítulos nuevos. Durante el verano los espectadores de series quedan algo abandonados.

Sería deseable, no obstante, que las cadenas mantuvieran actualizadas al minuto (como hacen con la actualidad periodística) las informaciones referentes a horarios y días de emisión de sus series.

\section{Quinto indicador: comunidad virtual}

Desde un punto de vista general podemos indicar que todas las webs espacio a la interactividad.

El interés del usuario por los diferentes aspectos y temas que rodean a las series de televisión hace que éstas se conviertan en un producto importante y que tenga que tenerse en cuenta por los directivos de las diferentes cadenas. de televisión dedican un importante
De manera específica, cabe señalar que Antena 3 presenta lo que denominan Zona 3, en la que el usuario puede registrarse como fan de las diferentes series. Además se aprecia una interrelación entre las diferentes herramientas dado que, en algunos casos, si se visualiza por ejemplo, algún "Encuentro digital", el usuario puede incluir algún comentario. Incluso algunas series tienen implementada una comunidad individual; tal es el caso de la serie El internado.

Telecinco brinda una opción específica para la participación del usuario desde el menú Gente. Al margen de las herramientas indicadas en la tabla anterior, también podrá recibir boletines de las diversas series.

Siguiendo esta misma mecánica se sitúa Cuatro donde, desde el menú Participa y en menor medida, Practica Cuatro, el usuario encuentra todas las oportunidades de intervenir. En esta web, resaltamos la

\begin{tabular}{|l|c|c|c|c|c|}
\hline \multicolumn{7}{|l|}{ Comunidad virtual } \\
\hline & Antena 3 & Telecinco & Cuatro & La sexta & TVE \\
\hline Foros & & $\mathrm{X}$ & $\mathrm{X}$ & $\mathrm{X}$ & \\
\hline Encuestas & $\mathrm{X}$ & $\mathrm{X}$ & $\mathrm{X}$ & & $\mathrm{X}$ \\
\hline Concursos/Sorteos & $\mathrm{X}$ & $\mathrm{X}$ & $\mathrm{X}$ & $\mathrm{X}$ & \\
\hline Juegos & $\mathrm{X}$ & $\mathrm{X}$ & $\mathrm{X}$ & $\mathrm{X}$ & \\
\hline Opinión/Comentarios & $\mathrm{X}$ & & $\mathrm{X}$ & $\mathrm{X}$ & $\mathrm{X}$ \\
\hline Redes sociales/Servicios web 2.0 & $\mathrm{X}$ & $\mathrm{X}$ & & & $\mathrm{X}$ \\
\hline Blogs & $\mathrm{X}$ & $\mathrm{X}$ & & & \\
\hline Encuentros digitales/Entrevistas & $\mathrm{X}$ & $\mathrm{X}$ & & $\mathrm{X}$ & $\mathrm{X}$ \\
\hline
\end{tabular}

Tabla 1. Opciones de interactividad en las series de televisión en internet 


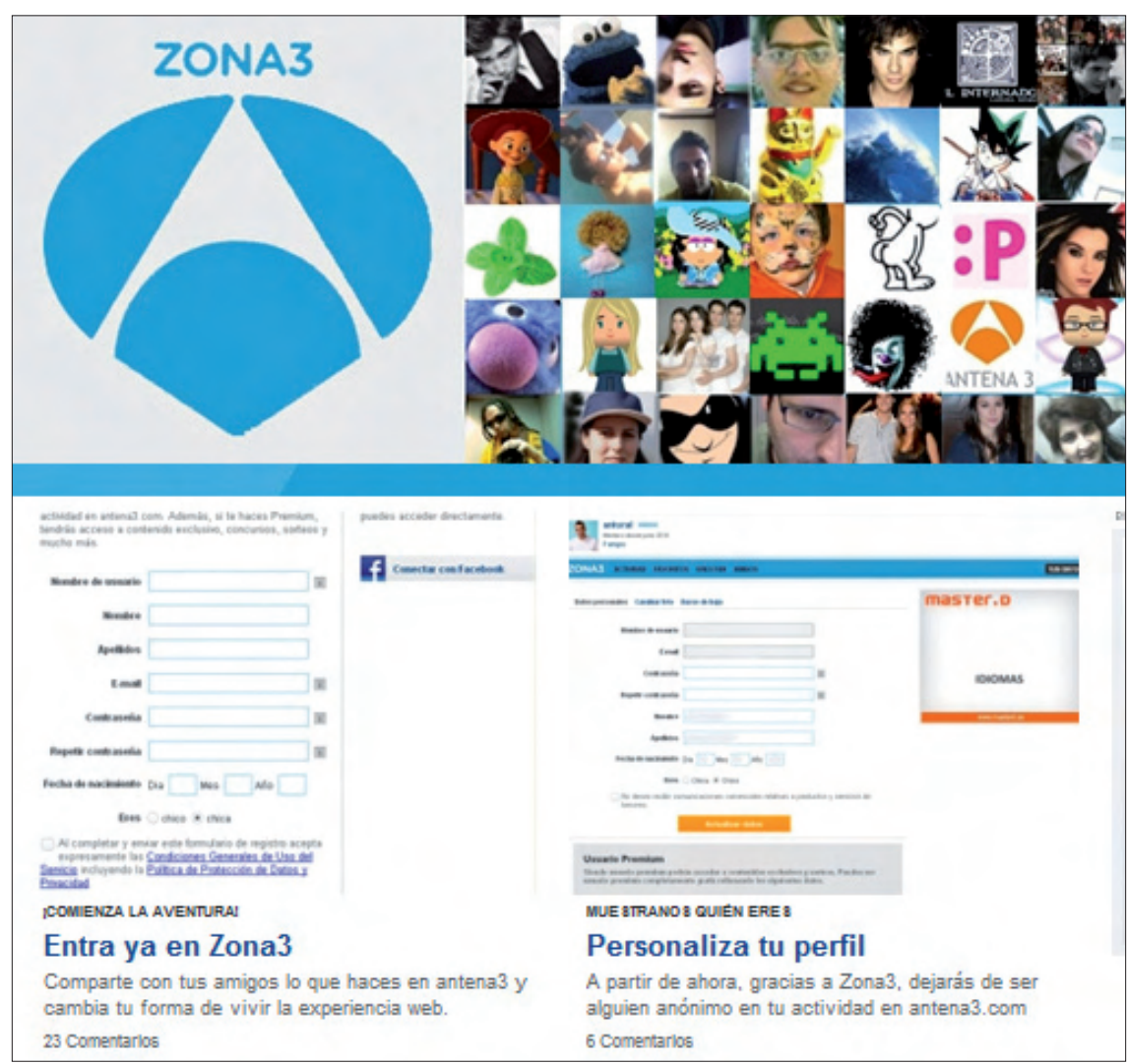

Figura 4. La comunidad Zona 3, de Antena 3 TV, http://www.antena3.com/zona3/

opción Juegos ya que se diferencia en cierta manera de los casos anteriores. Tal es el caso, por ejemplo, de la presentación de un Trivial sobre las diferentes series, idea basada en el formato original.

En esta misma línea, también destacamos esta opción participativa en La sexta por la vinculación de algunos juegos con las series, como los relacionados con El mentalista (CBS, 2008) donde el usuario tiene que resolver enigmas, ordenar elementos, etc. Por otra parte, aunque el usuario que accede encontrará el menú "Entrevistas digitales" cabe mencionar que esta opción no está disponible para las series, solamente para programas y noticias. Por este motivo no se ha señalado en la tabla.

En TVE se aprovecha la herramienta del chat de Facebook para convocar a una charla a los usuarios de la web. Mientras dura la emisión simultánea del capítulo (en la web y en el televisor), los espectadores/ internautas pueden ir haciendo sus comentarios en la página correspondiente.

\section{sing}

Sexto indicador: merchandi-

En España, contrariamente a lo que ocurre en Estados Unidos, las webs no proveen merchandising propio de los productos de la serie. Sí podemos encontrar los dvds de las series, algún libro y alguna prenda de vestir (camisetas, gorras), pero no está tan desarrollado como en las páginas oficiales. Algunos ejemplos de los productos encontrados son: TVE (dvd y libro de Herederos); Cuatro (dvd, cd de House y camisetas de Anatomía de Grey); y Telecinco (videojuego de $C S I$, -este juego es para comprar, no como los mencionados en el punto

$$
\begin{aligned}
& \text { “Los sitios web de } \\
& \text { series de televisión se } \\
& \text { caracterizan por una } \\
& \text { mayor permanencia del } \\
& \text { material para uso } \\
& \text { y disfrute del usuario" }
\end{aligned}
$$

5-; y dvds de series tales como Acusados, Aída, CSI, Hermanos y detectives, El pacto, etc.).

En cambio resulta curioso que exista un enlace denominado gifts donde, en lugar de encontrar regalos, es decir, una tienda para comprar cosas que regalar, encontramos salvapantallas (lo que en otras webs tradicionalmente se llama extras).

\section{Séptimo indicador: interacción}

Las páginas de las series deberían facilitar un correo para poder aportar comentarios o hacer preguntas sobre las series en general, $\mathrm{y}$, a su vez, cada una de las páginas de las series debería proporcionar un correo. Las cadenas optan por no mantener un correo electrónico específico y la comunicación con el usuario se limita a los foros y chats o a la posibilidad de participar en encuentros digitales.

\section{"Facilitaría la navegación al usuario (y potencial espectador) diferenciar claramente las series de otros formatos"}

\section{Conclusiones}

En primer lugar queremos señalar que el método de evaluación utilizado para este análisis es extrapolable a otros productos televisivos como son los programas de entretenimiento.

Facilitaría la navegación al usuario (y potencial espectador) diferenciar claramente las series de otros formatos ya que puede llevarle a engaño si se considera el mismo género a productos tan dispares como La isla de los nominados o El mentalista. Como decíamos al principio, la precisión terminológica es importante para que el espectador sepa a qué producto se enfrenta. Si ya des- 
de la promoción de la página web se confunden los formatos, el espectador puede prescindir del sitio web como futura fuente de información.

Si atendemos a la actualización, factor imprescindible cuando hablamos de una web, llama la atención la falta de actualización en las páginas de algunas series. Tal es el caso de Life, en cuya página aparecen datos de temporadas pasadas, e informaciones respecto a horarios y días de emisión que ya no son ciertas. Contrasta este hecho con la cantidad de información y lo actualizado de la misma en lo referido a otros formatos o a la información de actualidad.

Al analizar el estilo del sitio de cada serie (dentro de la web de una misma cadena) hay que señalar también la falta de uniformidad a la hora de planificar los diferentes sitios web corporativos y cada página de series en cada uno de ellos. Este hecho provoca una cierta desorientación cuando pasamos de una serie a otra.

\section{"La cantidad y calidad de la información que ofrecen las webs de las televisiones en España resulta insuficiente e imprecisa"}

Por último, en cuanto a la permanencia de los productos informativos referidos a las series, a diferencia de lo que ocurre con otros materiales dentro de un sitio web (por ejemplo, con los documentos de actualidad tales como noticias, fotografías, vídeos, etc.), existe una mayor permanencia del material que aquí se ha analizado para uso y disfrute del usuario.

Como conclusión final podemos señalar que la cantidad y la ca- lidad de la información que ofrecen las webs de las cadenas de televisión en España resulta insuficiente e imprecisa. Salvo algunas series de producción propia, o alguna con elevadas cifras de audiencia, no es extraño encontrar datos anticuados y escasos. La mezcla de formatos, los datos de series que ya no se emiten y otros factores analizados hacen que las páginas de series sean mejorables.

\section{Notas}

1. Para el estudio de la relación entre la televisión y la Red se recomienda el libro: Noam, Eli; Groebel, Jo; Gerbarg, Darcy (eds.). Internet television. Mahwah, NJ: Laurence Erlbaum, 2004. ISBN 0-8058-4306-X.

2. Jiménez, Maria-Àngels; Fuentes, MariaEulàlia; González, Alfons. "El profesional de la información ante el reto de los medios de comunicación digitales: el caso de la prensa". Scire: Representación y organización del conocimiento, 2002, v. 8, n. 2, pp. 141-152. Los autores analizan la importancia creciente de internet para los medios de comunicación de masas; aunque el fragmento que sigue se aplica a la prensa, es perfectamente aplicable a las cadenas de televisión: "Todo este universo de opciones informativas ha incorporado una amplia gama de servicios de valor añadido (SVA) que ha enriquecido y modificado la concepción tradicional de los medios de comunicación. [...] Por SVA entendemos aquellos servicios que se basan en el uso de las tecnologías y en el grado de interactividad que ofrece la Red para proporcionar al lector utilidades y fuentes de información que no están al alcance de los diarios convencionales por las limitaciones tecnológicas intrínsecas de sus versiones impresas".

http://ibersid.eu/ojs/index.php/scire/article/ download/1176/1158

3. El auge experimentado por las series de televisión fue estudiado por García-De-Castro, Mario. "Propiedades de la hegemonía de la ficción televisiva doméstica en España entre 19952000". Revista ZER, 2003, n. 14.

http://www.ehu.es/zer/zer14/propiedades14.htm

4. Stewart, Larry. La empresa de medición de audiencias Nielsen elaboró en el año 2000 un ranking con los programas más vistos entre 1961 y 2000. El número 1 de la lista es para el episodio final de la serie estadounidense $M * A * S * H$. http://articles.latimes.com/2001/jan/27/sports/ sp-17704

5. Se recomienda la lectura del análisis publicado sobre la serie Médico de familia: Herrero, Mónica; Diego, Patricia. "Do family television series travel well? A Spanish case: Médico de familia". Journal of Spanish language media, 2009, v. 2, pp. 142-154

6. Resulta interesante el trabajo abordado por Pacheco-Barrio sobre esta serie, una de las más galardonadas en la historia de la televisión. Pacheco-Barrio, Manuel A. "La reciente historia de España en la ficción televisiva". Mediaciones sociales, 2009, n. 4, pp. 225-246.

http://revistas.ucm.es/inf/19890494/articulos/ MESO0909120225A.PDF

7. Para el análisis de webs, junto con los trabajos de Lluís Codina, también resulta interesante el siguiente: Jiménez-Piano, Marina; Ortiz-Repiso, Virginia. Evaluación y calidad de sedes web. Gijón: Trea, 2007. ISBN 978-84-9704-318-2.

8. Spoiler es un término usado en series de televisión, procedente de la jerga empleada por los usuarios de los grupos de UseNet. Deriva del verbo inglés to spoil que significa arruinar, echar a perder.

\section{Bibliografía citada}

Codina, Lluís. "Evaluación de calidad en sitios web: proyectos de estudios sectoriales y realización de auditorías". En: Jornades Catalanes d'Informació i Documentació, 2004.

Codina, Lluís; Aubia, Laia; Sánchez, Noemí. "Propuesta nuclear de análisis de sitios web de televisión", 2008.

http://www.lluiscodina.com/analisistv2008.doc

Dafonte-Gómez, Alberto. "Reflexiones teóricas en torno al concepto de género y a las dificultades que plantea su aplicación en la investigación sobre contenidos televisivos". Comunicación y sociedad, 2010, v. XXIII, n. 1, pp. 121-152.

http://www.unav.es/fcom/comunicaciony sociedad/documentos/pdf/20100602124347.pdf

García-De-Castro, Mario. "Los movimientos de renovación en series televisivas españolas". Comunicar, 2008, v. XV, n. 30, p. 152. http://redalyc.uaemex.mx/pdf/158/15811864023. $p d f$

Gómez-Martínez, Pedro; García-García, Francisco. El guión en las series televisivas: formatos de ficción y presentación de proyectos, pp. 169-177. Madrid: Univ Francisco de Vitoria, 2010. ISBN 978-84-8955-256-2.

Gordillo, Inmaculada. La hipertelevisión: géneros y formatos, pp. 42-44. Quito: Ediciones Ciespal, 2009. ISBN 978-99-7855-071-7.

Jiménez, Maria-Àngels; Fuentes, MariaEulàlia; González, Alfons. "Los archivos audiovisuales en los medios de comunicación digital". Scire: Representación y organización del conocimiento, 2003, v. 9, n. 2, pp. 99-110.

http://ibersid.eu/ojs/index.php/scire/article/ view/1469/1447

Elena De-la-Cuadra-Colmenares. Facultad de Ciencias de la Información, Universidad Complutense de Madrid.

ecuadra@ccinf.ucm.es

María-Victoria Nuño-Moral. Facultad de Biblioteconomía y Documentación, Universidad de Extremadura, 06071 Badajoz. mvnunmor@alcazaba.unex.es 